\title{
Fuentes femeninas y masculinas en la cobertura del feminicidio en la prensa mexicana
}

\section{Male and female sources in the coverage of femicide in the Mexican press}

\section{Fontes femininas e masculinas na cobertura do feminicídio na imprensa mexicana}

Elizabeth Tiscareño-García, Tecnológico de Monterrey, Campus Monterrey, México (A01470053@exatec.tec.mx)

Santiago Gallur Santorum, Instituto Tecnológico de Santo Domingo (INTEC), Santo Domingo, República Dominicana (santiago.gallur@intec.edu.do)

Oscar Mario Miranda-Villanueva, Tecnológico de Monterrey, Campus Estado de México, México (oscar.miranda@tec.mx)

RESUMEN | En este estudio se investigan las fuentes periodísticas utilizadas por la prensa mexicana en noticias sobre el feminicidio. El objetivo es analizar las dinámicas de la selección de esas fuentes con relación al género y su oficialidad. La investigación de corte cuantitativo estudia el contenido sobre el feminicidio y la violencia de género en cuatro periódicos mexicanos de tirada nacional: La Jornada, Reforma, El Universal y Milenio durante 2017. De 1324 textos, se analizaron 2091 fuentes. Estas se clasificaron con un modelo preestablecido: fuentes oficiales que aceptan la versión oficial respecto de la información sobre hechos de feminicidio, oficiales que rechazan la versión oficial, protagonistas de los hechos, víctimas de las situaciones y especialistas, expertas o con voluntad de expresarse. El resultado mostró que las fuentes femeninas cuestionan la versión oficial, mientras que las masculinas la apoyan y la sostienen. Asimismo, las fuentes femeninas tuvieron mayor presencia como expertas. Las fuentes femeninas construyen el discurso crítico sobre el feminicidio, mientras que las masculinas lo sostienen con versiones oficiales. La investigación constata un elevado nivel de discriminación hacia las mujeres como fuentes de información oficial en el tema del feminicidio en México. De hecho, se puede afirmar que el género es una categoría que los medios de comunicación usan para discriminar las fuentes válidas en la construcción del hecho noticioso.

PALABRAS CLAVE: fuente; género; prensa; periódico; feminicidio; México. 
ABSTRACT | This study investigates the journalistic sources used in the Mexican press in news about feminicide. The aim is to analyze the dynamics of the sources' selection regarding gender and its official status (officiality). The research is quantitative and studies the content on feminicide and gender violence in four newspapers of national circulation: La Jornada, Reforma, El Universal and Milenio during 2017. Of 1324 news pieces emerged 2091 sources for analysis. These were classified with a pre-established model: official sources that accept the official version regarding the information on the facts offemicide, officials who reject the official version, protagonists of the events, victims of the situations and specialists, experts or willing to express themselves. The result shows that the female sources question the official version, while the male ones support and sustain it; female sources also have greater presence as experts regarding femicide. The female sources build the critical discourse on femicide, while male sources support the hegemonic discourse. The research confirms a high level of discrimination against women as official sources of information about feminicide in Mexico. Gender is a category that the media use to discriminate the valid sources in the construction of the news event.

KEYWORDS: sources; gender; press; newspaper; femicide; Mexico.

RESUMO | Este estudo investiga as fontes jornalísticas utilizadas pela imprensa mexicana em textos relacionados ao feminicídio. O objetivo é a análise da dinâmica de seleção dessas fontes em relação ao gênero e sua oficialização. A pesquisa quantitativa estuda o conteúdo sobre o feminicídioe a violência de gênero em quatro jornais mexicanos de circulação nacional: La Jornada, Reforma, ElUniversal e Milenio durante 2017. De 1.324 textos foram analisadas 2.091 fontes. As fontes foram classificadas com um modelo pré-estabelecido: funcionários que aceitam a versão oficial respeito de informações sobre atos de feminicídio, funcionários que rejeitam a versão oficial, protagonistas dos acontecimentos, vítimas das situações e especialistas, expertos ou com vontade de se expressar. O resultado mostra que as fontes femininas questionam a versão oficial, enquanto as fontes masculinas apoiam e sustentam. Além disso, as fontes femininas têm maior presença como especialistas. As fontes femininas constroem o discurso crítico sobre o feminicídio, enquanto as fontes masculinas sustentam um discurso baseado na versão oficial. A pesquisa revela um alto nível de discriminação contra as mulheres como fontes de informação oficiais sobre o feminicídio no México. De fato, pode-se afirmar que o gênero é uma categoria que a mídia utiliza para discriminar as fontes válidas na construção do acontecimento noticioso.

PALAVRAS-CHAVE: fontes; gênero; imprensa; jornal; feminicídio; México. 


\section{INTRODUCCIÓN}

Durante el año 2017, las autoridades mexicanas y la cobertura mediática hicieron énfasis en el incremento de los casos de feminicidio (Xantomila, 2020). Desde entonces, la estadística oficial muestra un alza constante de los feminicidios en México (Tiscareño-García, 2021; Salazar \& de la Garza, 2020), pese a cumplirse en esa fecha una década de la promulgación de la Ley General de Acceso de las Mujeres a una Vida Libre de Violencia (LGAMVLV) con la que el Estado pretendió hacerle frente a este problema (Jasso \& González, 2018; Tagle Martínez, 2017; Bernal \& Flores, 2018). En 2017 hubo 741 víctimas de feminicidio, una cifra mayor que la de 2016, cuando se registraron 605 feminicidios (SESNSP, 2020), y que la de 2015 (411 asesinatos). Es decir, en dos años el incremento fue de 330 casos (44,5\%).

El feminicidio comprende el asesinato de una mujer, o la tentativa, a manos de hombre por razones de género, en algunos casos bajo la anuencia social y la permisividad de las estructuras políticas y legales (Bejarano, 2014; Monárrez, 2019; Tiscareño-García \& Miranda Villanueva, 2020). En 1990, Caputi y Russell definieron el feminicidio como el asesinato de una mujer a manos de un hombre por el hecho de ser mujer. También representa el extremo de terror antifemenino a través de abusos verbales y físicos (Radford \& Russell, 1992; Monárrez, 2010). La ley mexicana lo considera como la forma extrema de violencia de género en contra de las mujeres, violentando sus derechos tanto en el ámbito público como en el privado (Ley General de Acceso de las Mujeres a una Vida Libre de Violencia, 2007).

Como parte de la construcción de los textos periodísticos, las fuentes informativas son consideradas la sustancia del periodismo (Lippmann, 1965; Shoemaker \& Reese, 2014). Aunque en las narraciones noticiosas se busca evitar la intrusión manifiesta de los valores personales del periodista, la literatura acerca de las fuentes coincide en que los trabajadores de los medios tienden a generar compromisos con algunas de estas (Berkowitz, 2009). Es por ello que se considera que el uso constante de las mismas fuentes monopoliza la información y hace que el periodista se aleje de las fuentes alternativas. El estudio de la selección de las fuentes informativas en la construcción de los textos periodísticos es un tema que se presta para su investigación en México, en especial en casos como el del feminicidio. Es por ello que aquí se analizan las fuentes informativas a las que recurrieron las y los periodistas mexicanos de la prensa en la cobertura noticiosa sobre casos de feminicidios durante 2017, con relación al género y su oficialidad.

Se busca conocer el rol de las fuentes informativas en los textos periodísticos sobre feminicidio en la prensa mexicana con relación al género, establecer en qué tipo de fuentes se recurre más a hombres o mujeres y a qué discurso responden más. 


\section{Revisión de la literatura}

Distintos estudios analizan cómo las fuentes, que son el origen de la información periodística, tienen un impacto relevante en la construcción de las noticias. Las perspectivas más comunes para estudiar las fuentes están relacionadas con las siguientes prácticas, de acuerdo con distintos autores:

1. Los reporteros aceptan las versiones de las fuentes autorizadas y sus reglas de divulgación para formar alianzas tácitas (Sigal, 1986).

2. Las fuentes poderosas usan su influencia para crear noticias que les favorecen (Gans, 2004).

3. Las fuentes privilegiadas facilitan buena información, hacen favores a los periódicos y esperan recibir algo a cambio (Grijelmo, 2002).

4. Ninguna fuente se comunica como un libre agente sin la influencia de la posición que tiene en el sistema sociocultural (Berlo, 1999).

5. Los periodistas recopilan datos autorizados y luego los presentan sin tomar partido explícitamente en el discurso pero, al final, están en deuda por la materia prima que les proporcionaron los entrevistados (Herman \& Chomsky, 1988).

6. Los periodistas incluyen y no desafían las interpretaciones de las fuentes oficiales porque son representantes de su comunidad y son un reflejo de los mismos reporteros (Breed, 1958).

No obstante, el poder no solo se ejerce mediante la proactividad intencional de las fuentes. También sucede entre los supuestos dados por sentado; es decir, cuando los periodístas no investigan a fondo y se quedan con lo que se les dice, ya que la dependencia del periodista de las fuentes puede provocar una desafección de la realidad y una visión sesgada. Ello debido a que solo se le proporciona la información que tienen que saber, además de enfrentar varias presiones antes de publicar una noticia (Manning, 2013, Ortega \& Humanes, 2000; Carpenter, 2008; Tuchman, 1973; Sigal, 1986). Las fuentes otorgan a los periodistas autonomía en su trabajo, debido a que estas constituyen su patrimonio personal; por lo tanto, las fuentes son consideradas como un recurso imprescindible para construir la noticia y conferirle credibilidad (Bezunartea,1988; Diezhandino, 2007; Gómez, 2016; Lozano, 2007; Menéndez, 2014). En ese contexto, la literatura muestra que las noticias van cargadas de intereses individuales y colectivos de quienes generan la información.

La fuente está representada en aquella persona a la que los reporteros recurren para obtener la información, por lo que frecuentemente son funcionarios o expertos 
relacionados con las instituciones centrales de la sociedad (Berkowitz, 2009). Shoemaker y Reese (2014) plantearon la problemática de la dependencia de cada tipo de publicación de las fuentes externas como los comunicados, las organizaciones y los individuos. Retegui (2017) destaca la importancia que tiene el estudio de las fuentes cuando están representadas a través de diversos tipos de documentos. En este artículo, la fuente es aquella persona que proporciona información con valor noticioso con la que el periodista elabora un texto informativo. Esta puede ser de distinto tipo: especialista, experto, funcionario, ciudadano o recursos como comunicados, boletines, estudios, investigaciones y fuentes anónimas.

En relación con los casos de estudio de la literatura (Bystrom, 2004; Freedman et al., 2004; Powers \& Fico, 1994; Van der Pas \& Aaldering, 2020), se muestra un cuestionamiento explícito al poder de las fuentes y la forma en la que se producen los textos informativos, y se reitera que los medios de comunicación tienden a recurrir a las fuentes oficiales. Lo anterior produce una homogeneización en los contenidos en la prensa que va en contra de la narrativa de la sociedad de la información, la que según Gallur y García (2019) promueve el equilibrio entre la participación del género. Entre esos estudios están los que exploran la diversidad de fuentes por género, cuyos hallazgos muestran el predominio de las fuentes masculinas (Freedman et al., 2004).

Además, los estudios sobre las fuentes se enfocan principalmente en comparativos de mujeres y hombres desde la perspectiva política y del espacio que ocupan en los medios de comunicación (Bystrom, 2004; Freedman et al., 2004; Van der Pas \& Aaldering, 2020). Powers y Fico (1994) explican que los medios de comunicación tradicionales citan más a las fuentes oficiales que ocupan posiciones de poder, con un predominio de fuentes masculinas, que aparecen con mayor frecuencia en las secciones de negocios y deportes. Sin embargo, estos investigadores determinan que los periodistas no seleccionan conscientemente las fuentes en función del género (Powers \& Fico, 1994), sino por diversos factores, como los roles sociales.

Por otra parte, están los estudios de grandes proporciones (GMMP, 2021; LenRíos et al., 2005; Pew Research Center, 2005; Tamarit et al., 2011), cuyos resultados son coincidentes y reiterativos al mostrar que los medios de comunicación discriminan a la mujer como fuente. Tras analizar 16.800 noticias en periódicos de Estados Unidos, el Pew Research Center (2005) determinó que más de tres cuartos de las historias usaron fuentes masculinas y solo un tercio contiene una sola fuente femenina. Esos datos sugieren que la representación de las mujeres en las noticias está alejada de reflejar el real rol de la mujer en la sociedad.

Desde esa perspectiva, el Proyecto de Monitoreo Global de Medios 2020 (GMMP, 2021) dio a conocer que de las personas entrevistadas, escuchadas, vistas, o sobre 
quienes se ha escrito en la prensa, radio y televisión solo $25 \%$ eran mujeres, contra $75 \%$ de hombres. Especialmente en la prensa escrita hay un $26 \%$ representado por mujeres y $74 \%$ por hombres. Desde el mismo ángulo, Tamarit y sus colegas (2011) señalan que, de 12.551 textos informativos recogidos en la prensa española, en 1603 las mujeres aparecieron como protagonistas de la información, lo que representó un $12,77 \%$. Además, la mayoría de las noticias que tienen a mujeres como protagonistas no había sido firmada, por lo que la información se obtuvo de ruedas de prensa o comunicados (Tamarit et al., 2011). Los estudios antes mencionados infieren que la masculinización de las fuentes es un problema de orden mundial, en el que prevalecen culturas semejantes al momento de seleccionar las fuentes.

Otros trabajos sobre el estudio de las fuentes se enfocan en establecer patrones culturales dominantes en las formas en las que se producen las noticias, con énfasis en la presencia de voces masculinas y en la invisibilización de las femeninas. En la investigación de Len-Ríos y sus colegas (2005) se estudió la representación de las mujeres en los periódicos y se comparó con las percepciones del personal de noticias y los lectores para explorar el concepto de hegemonía cultural masculina. La primera parte mostró que los hombres aparecen más que las mujeres en historias y fotografías. Los autores establecieron que los periódicos estudiados reflejan la hegemonía cultural masculina predominante en la cultura estadounidense, ya que alrededor de las tres cuartas partes de las personas en las noticias eran hombres y dos tercios de las fotos mostraban hombres.

Trabajos como el de Lagos (2008) sobre violencia de género o feminicidio en la prensa reflejan que las fuentes se incluyen de manera marginal y acrítica en casos de feminicidio, y se acude a fuentes cruciales como los tribunales o la policía. De esa manera, el feminicidio se presenta a través de conceptos como el policial, el penal y el judicial. En esos casos, los registros oficiales podrían culpabilizar a la víctima si hubo incidentes en los que esta no quiso dar su declaración o retiró los cargos pese a los esfuerzos policíacos (Taylor, 2009). Por su parte, las fuentes de las instituciones de igualdad y de los movimientos organizados son tomadas en cuenta desde las acciones de protesta por la muerte de una mujer (Menéndez, 2014).

La literatura también advierte que en la producción de la noticia están presentes el género, la etnia, la educación y la orientación sexual de quien escribe (Berkowitz \& Liu, 2014). Así, la masculinización de los medios se ve reflejada también en las plantillas laborales (Danés, 2017; Niemi \& Pitkanen, 2016; Tiscareño-García \& Miranda-Villanueva, 2020). Asimismo, los estudios muestran que, por lo general, la inmediatez implica que los medios cubran los mismos hechos y acudan a las mismas fuentes, lo que genera una uniformidad en sus contenidos. También se vuelve una costumbre el uso de datos similares a los de la competencia y cómo 
en ese proceso se discriminan las voces femeninas y se hace a un lado el balance y la confrontación de ideas.

El desequilibrio en las oportunidades de las mujeres en diversos espacios sociales es observado desde la óptica legal como discriminación, que implica impedir y anular el reconocimiento o los ejercicios de los derechos y la real igualdad de oportunidades de las personas (Ley General para la Igualdad entre Hombres y Mujeres, 2021). La exclusión está inserta en la discriminación y responde a un proceso multidimensional y multicausal que concierne a distintos factores dentro del ámbito jurídico, económico, social e individual (Rizo, 2012). Tanto la discriminación como la exclusión se traducen en falta de espacios para que la mujer obtenga igualdad y agencia de manera real en temáticas de interés social.

Una de las investigaciones más recientes sobre las fuentes informativas y el tema del feminicidio es la de Gallur y García (2019). Este estudio cuantitativo se centra en el uso de las fuentes según el género, y plantea que en los medios de comunicación persiste el discurso hegemónico. Específicamente, se analiza la cobertura del feminicidio en Ciudad Juárez realizada por la agencia de noticias de género mexicana Cimacnoticias (CIMAC) entre 1999 y 2015. Dicho análisis se centró en el estudio de 2712 fuentes utilizadas para elaborar 1284 noticias. Los resultados señalan que, a medida que disminuye el nivel de oficialidad, aumenta la presencia de las mujeres como fuentes en las noticias.

Con ese contexto, esta investigación tiene como punto de partida las siguientes premisas:

1. La prensa nacional mexicana, independientemente de su postura ideológica y de sus prácticas para seleccionar las fuentes informativas, generalmente recurre a fuentes oficiales masculinas.

2. Las fuentes oficiales masculinas disminuyen la perspectiva crítica.

3. Las notas relacionadas con los casos de feminicidio en la prensa nacional mexicana reducen la perspectiva crítica.

\section{METODOLOGÍA}

El universo de la muestra abarcó los textos periodísticos relacionados con el feminicidio y la violencia de género publicados del 1 de enero al 31 de diciembre de 2017 en los periódicos La Jornada, Reforma, El Universal y Milenio. Se decidió este periodo porque comprende el año en que las autoridades y las organizaciones civiles hicieron énfasis públicamente en el incremento de las estadísticas de feminicidios en México. 
Este artículo forma parte de una investigación más amplia que estudia el lenguaje en la prensa mexicana sobre feminicidio; el estudio en general contempla 1679 textos periodísticos (tabla 1). La unidad de análisis se estableció como los textos noticiosos que abordan el tema de la violencia de género o feminicidio y que presentaron la problematización en distintos contextos. Por ello, quedaron fuera los textos en los que las fuentes no aportaban al debate y en donde las palabras de búsqueda solo se enunciaban en el texto y no se profundizaba en ese tema. Por lo mismo, el censo de 1679 se redujo a 1324 textos: La Jornada (18,5\%), Reforma (16,5\%), El Universal (33\%) y Milenio (32\%). De esos 1324 se desprendieron 2091 fuentes (tabla 1).

Se estudiaron las noticias sobre el feminicidio en la prensa mexicana en estos cuatro periódicos por considerarse de referencia y tener tiraje nacional. Sobre el espectro ideológico hay que considerar que los medios que se sitúan a la derecha se guían como voceros de los intereses de las grandes empresas y se rigen por los valores de ese grupo, como en el caso de Reforma (Durán, 2009; Álvarez-Monsiváis, 2020). Salazar y sus colegas (2019), no obstante, lo describen como híbrido, al considerarlo cívico y capitalista. En tanto, aquellos medios que se sitúan al centro están definidos por la pluralidad de ideas y una ideología moderada (Bautista, 2013); aquí entran El Universal y Milenio. El primero comenzó como un diario tradicionalista, sin contradecir los intereses del gobierno (Rodelo \& Muñiz, 2016). Milenio surgió con la coyuntura de las campañas electorales de 2000 (Salgado, 2005). La prensa de izquierda utiliza el discurso crítico, entabla denuncias hacia el poder político y las élites de poder (Salgado, 2005); en ese espectro se posiciona La Jornada, como asociación cooperativa, sin nexos con grandes conglomerados, con una actitud anti-régimen (Rodelo \& Muñiz, 2016).

La metodología empleada en esta investigación es cuantitativa. Se utilizó el análisis de contenido como herramienta de análisis de estudio y de análisis de comunicación: sistemático, objetivo y cuantitativo, que mide determinadas variables (Wimmer \& Dominick, 2011).

La información de los cuatro periódicos se extrajo de manera digital. En este caso, se recolectó la información de todos los textos incluidos en los criterios del universo o población, es decir, aquellos textos que contenían los componentes de feminicidio, homicidio, muerta, crimen, mujer, niña y joven. Para Milenio y La Jornada las noticias se seleccionaron del portal de cada medio. En el caso de El Universal y Reforma se hizo con la base de datos ProQuest, a la que se accedió a través de la biblioteca digital del Tecnológico de Monterrey. 


\begin{tabular}{cccccc} 
Temática & La Jornada & Reforma & El Universal & Milenio & Total \\
\hline Feminicidio y violencia & 348 & 257 & 549 & 525 & 1679 \\
de género (general) & $20,7 \%$ & $15,3 \%$ & $32,7 \%$ & $31,3 \%$ & $100 \%$ \\
\hline $\begin{array}{c}\text { Feminicidio y violencia } \\
\text { de género (problemática) }\end{array}$ & 246 & 217 & 439 & 422 & 1324 \\
\hline Total de fuentes & 530 & $16,5 \%$ & $33 \%$ & $32 \%$ & $100 \%$ \\
\hline & $25,35 \%$ & $16,3 \%$ & $31,7 \%$ & $26,65 \%$ & $100 \%$ \\
\hline
\end{tabular}

Tabla 1. Desglose de textos y fuentes por periódico

Fuente: Elaboración propia.

En la búsqueda avanzada para el diario Reforma se utilizó la identificación de la publicación (PUBID) (32.652) AND (Mujer OR niña OR joven) AND (asesinada OR homicidio OR muerta OR feminicidio OR crimen). En El Universal, la búsqueda fue PUBID (47.134) AND (Mujer OR niña OR joven) AND (asesinada OR homicidio OR muerta OR feminicidio OR crimen). En el caso de La Jornada para el acceso se empleó un vínculo del portal del periódico que llevaba al buscador de Google a través de la página de la Universidad Nacional Autónoma de México (UNAM). Los criterios que se emplearon fueron: (ene.2017) AND (Mujer OR niña OR Joven) AND (asesinada OR homicidio OR muerta OR feminicidio OR crimen). Respecto de Milenio, la búsqueda se hizo directamente en la página del periódico y se utilizaron las palabras: (enero.2017 Feminicidio); para Milenio y La Jornada, las búsquedas se hicieron por cada mes. Se aplicaron tres métodos distintos de búsqueda dependiendo del acceso de cada periódico.

Con base en un esquema preestablecido del estudio de las fuentes se elaboró en Excel un modelo de codificación en el que se analizaron los 1324 textos. El análisis se desarrolló en dos partes; en la primera, se cuantificaron los textos periodísticos y la cantidad de fuentes: quién redacta la información; la clasificación del texto, y el número de fuentes. Las variables del género periodístico eran: noticia, resumen, reportaje, artículo de opinión y otros, como entrevistas y gráficos, mientras que para el género del periodista fueron: reportero, reportera, ambos, y el periódico cuando la firma era de la redacción, staff o del periódico. Acerca del número de fuentes se establecieron desde 0 a 12 fuentes, que fue el rango que presentaron los textos.

En la segunda parte, se estudiaron las características de las fuentes utilizando el modelo ya trabajado por Gallur y García (2019), que preestablece cinco categorías:

4. Fuentesoficialesqueapoyanla versión oficial.Sonlasfuentesinstitucionalizadas, forman parte del sistema gubernamental o están relacionadas con él; este tipo de fuentes difunden y sostienen la versión oficial. Son funcionarios de instituciones públicas de los tres poderes de gobierno, principalmente del área 
de seguridad y justicia, así como de organismos nacionales e internacionales institucionalizados. Las fuentes pueden ser femeninas, masculinas y las que aluden a fuentes no identificadas o a algún tipo de documento.

5. Fuentes oficiales que contradicen la versión oficial. Es el mismo tipo de fuentes que la clasificación anterior, pero en este caso cuestionan o no están de acuerdo con la versión oficial.

6. Protagonistas de los hechos. Se trata de las personas involucradas directamente en el caso de feminicidio. La víctima y el victimario o presunto victimario.

7. Víctimas de las situaciones. Son mujeres que fallecen o sobreviven al presenciar o defender a una víctima de feminicidio, sufren ataques similares al de la persona que defienden.

8. Expertos y personas con información sobre el tema y voluntad de expresarla. Son fuentes que ofrecen información con relación al feminicidio como especialistas o expertas, que están fuera del ámbito oficial, o aquellas cercanas al hecho como familiares y testigos. Son clasificadas como: femeninas, masculinas y aquellas que no tienen un vocero específico, pero asignan la información a una asociación o instancia o mencionan algún tipo de documento.

La codificación de las piezas informativas la hizo una sola persona, quien fue entrenada con los criterios de evaluación de cada variable para mantener una postura objetiva al momento de codificar la información.

\section{RESULTADOS}

\section{Aspectos generales del estudio de las fuentes informativas}

De los 1324 textos se obtuvieron 2091 fuentes, de las cuales 778 (37\%) promueven la versión oficial. 332 (16\%) fueron fuentes oficiales que rechazan la versión oficial, mientras que las fuentes protagonistas de los hechos corresponden a 0,05\% (una fuente). Aquellas víctimas de las situaciones tuvieron un $0 \%$, y se contabilizaron 980 (46,95\%) fuentes especialistas, expertas o con voluntad de expresarse (tabla 2).

De los cuatro periódicos estudiados en el desglose por género y periódico, las voces femeninas fueron 668 (32\%); la proporción fue superior a las masculinas (447, $21,5 \%$ ), mientras que los documentos o fuentes anónimas comprendieron 976 fuentes $(46,5 \%)$ (tabla 3). De lo anterior se establece de forma general un comportamiento homogéneo por parte de los periódicos analizados, que al momento de seleccionar las fuentes para construir el texto acerca del feminicidio acuden principalmente a documentos, después a fuentes femeninas y, finalmente, a masculinas. 


\begin{tabular}{|c|c|c|c|c|c|}
\hline Fuentes/periódicos & La Jornada & Reforma & El Universal & Milenio & Total \\
\hline \multicolumn{6}{|c|}{ Fuentes oficiales que promueven la versión oficial $(778 ; 37 \%)$} \\
\hline Masculina & $\begin{array}{l}47 \\
9 \%\end{array}$ & $\begin{array}{c}37 \\
11 \%\end{array}$ & $\begin{array}{l}58 \\
9 \%\end{array}$ & $\begin{array}{c}55 \\
10 \%\end{array}$ & 197 \\
\hline Femenina & $\begin{array}{c}9 \\
2 \%\end{array}$ & $\begin{array}{l}17 \\
5 \%\end{array}$ & $\begin{array}{l}20 \\
3 \%\end{array}$ & $\begin{array}{l}43 \\
8 \%\end{array}$ & 89 \\
\hline $\begin{array}{l}\text { Documentos e } \\
\text { instituciones }\end{array}$ & $\begin{array}{c}85 \\
16 \%\end{array}$ & $\begin{array}{c}90 \\
26 \%\end{array}$ & $\begin{array}{l}195 \\
29 \%\end{array}$ & $\begin{array}{l}122 \\
22 \%\end{array}$ & 492 \\
\hline
\end{tabular}

Fuentes oficiales que rechazan la versión oficial (332; 16\%)

\begin{tabular}{cccccc}
\hline Masculina & 33 & 5 & 13 & 11 & 62 \\
& $6 \%$ & $1.5 \%$ & $2 \%$ & $2 \%$ & 109 \\
Femenina & 55 & 5 & 25 & 24 & $4 \%$ \\
\hline $\begin{array}{c}\text { Documentose } \\
\text { instituciones }\end{array}$ & $10 \%$ & $1.5 \%$ & $4 \%$ & $4 \%$ & \\
\hline
\end{tabular}

\begin{tabular}{cccccc}
\hline \multicolumn{7}{c}{ Fuentes protagonistas de los hechos (1; 0,05\%) } \\
\hline Masculina & 0 & 0 & 1 & 0 & \\
\hline Femenina & $0 \%$ & $0 \%$ & $0,15 \%$ & $0 \%$ & 1 \\
\hline Documentos & 0 & 0 & 0 & 0 & 0 \\
\hline
\end{tabular}

\begin{tabular}{cccccc}
\multicolumn{7}{c}{ Fuentes víctimas de las situaciones (0; 0\%) } \\
\hline Masculina & 0 & 0 & 0 & 0 & 0 \\
\hline Femenina & 0 & 0 & 0 & 0 & 0 \\
\hline Documentos & 0 & 0 & 0 & 0 & 0 \\
\hline Fuentes expertas, especialistas y personas con voluntad de expresarse (980; $\mathbf{4 6 , 9 5 \% )}$ \\
\hline Masculina & 27 & 50 & 66 & 44 & 187 \\
\hline Femenina & $5 \%$ & $15 \%$ & $10 \%$ & $8 \%$ & 470 \\
\hline Documentos & 112 & 75 & 150 & 133 & 323 \\
\hline Total & $21 \%$ & $22 \%$ & $23 \%$ & $24 \%$ & 2091 \\
\hline & $18 \%$ & $11 \%$ & $14,85 \%$ & $16 \%$ & $100 \%$ \\
\hline
\end{tabular}

Tabla 2. Desglose de categorías por fuente y periódico, 1324 textos, 2091 fuentes

Fuente: Elaboración propia. 


\begin{tabular}{cccccc} 
Fuentes & La Jornada & Reforma & El Universal & Milenio & Total \\
\hline Masculinas & 107 & 92 & 138 & 110 & 447 \\
& $5,1 \%$ & $4,4 \%$ & $6,6 \%$ & $5,4 \%$ & $21,5 \%$ \\
\hline Femeninas & 176 & 97 & 195 & 200 & 668 \\
\hline $\begin{array}{c}\text { Documentos o sin } \\
\text { identificar }\end{array}$ & $8,4 \%$ & $4,6 \%$ & $9,4 \%$ & $9,6 \%$ & $32 \%$ \\
\hline Total & $11,5 \%$ & 152 & 330 & 247 & 976 \\
& 530 & $34 \%$ & $16 \%$ & $12 \%$ & $46.5 \%$ \\
\hline & $25,35 \%$ & $16,3 \%$ & $31,7 \%$ & $26,65 \%$ & $100 \%$ \\
\hline
\end{tabular}

Tabla 3. Desglose de fuentes por género y por periódico

Fuente: Elaboración propia.

A partir de estos datos generales, en los siguientes párrafos se presenta el análisis correspondiente a cada premisa, por lo que se consideran aspectos específicos del análisis.

\section{Las fuentes oficiales en el tema del feminicidio en la prensa mexicana}

La primera premisa consistió en medir la recurrencia a fuentes oficiales masculinas por parte de la prensa nacional mexicana, independientemente de su postura ideológica. En la categorización de las 2091 fuentes informativas, 53\% correspondió a fuentes oficiales, que resultan de las fuentes que aceptan (778; $37 \%)$ y rechazan (332; 16\%) la versión oficial. Aunque el porcentaje de documentos, es decir, boletines, comunicados o fuentes sin identificar tuvo mayor frecuencia (58,5\%), el género del reportero pudo ser identificado en 457 noticias (41,5\%), con una mayor presencia masculina (tabla 4). Desde una perspectiva general, las fuentes femeninas ocuparon mayor espacio en los textos periodísticos, pero cuando se trata de la oficialidad de las fuentes, la presencia masculina fue más importante.

\begin{tabular}{ccc} 
Fuentes oficiales por género & Número de fuentes & Porcentaje \\
\hline Masculina & 259 & $23,5 \%$ \\
\hline Femenina & 198 & $18 \%$ \\
\hline Documentos & 653 & $58,5 \%$ \\
\hline Total & 1110 & $100 \%$ \\
\hline
\end{tabular}

Tabla 4. Desglose de las fuentes oficiales

Fuente: Elaboración propia. 


\section{Las fuentes oficiales que aceptan y rechazan la versión oficial}

En el planteamiento de la segunda premisa que considera que las fuentes oficiales masculinas disminuyen la perspectiva crítica, el análisis de tres de las categorías permitió conocer más acerca de este aspecto: las fuentes oficiales que aceptan la versión oficial, las fuentes oficiales que la rechazan y las fuentes especialistas, expertas o con voluntad de expresarse (tabla 2).

Las fuentes que aceptan y rechazan la versión oficial tuvieron mayor concentración de fuentes masculinas $(25,3 \%)$ que femeninas; cuando se trata de género identificable, al momento de construir el texto la o el periodista recurren más a voces masculinas que tienden a apoyar la versión oficial y en menor proporción a femeninas $(11,4 \%)$. Lo anterior se revierte cuando se trata de la categoría de las fuentes oficiales que rechazan o no están de acuerdo con la versión oficial acerca del feminicidio en la prensa mexicana. La cantidad de mujeres en desacuerdo con la versión oficial fue de $32,84 \%$, mientras que los hombres entrevistados estuvieron en el orden de 18,67\%. Eso pone en un plano más crítico la narrativa femenina que la masculina (tabla 5); por ende, las fuentes masculinas oficiales tienden a presentar un discurso menos crítico y solo permean la versión oficial.

La tercera categoría en el estudio de la perspectiva crítica acerca de las fuentes oficiales masculinas trata de voces de expertas, especialistas y personas con voluntad de expresarse (980 fuentes, $46,95 \%$ de las 2091). Las fuentes femeninas son más consultadas (470) que las masculinas (187). Este tipo de fuentes se comportó como una alternativa no oficial, mientras que la cantidad de masculinas fue $250 \%$ menor que las femeninas (tabla 2). Lo anterior evidencia que los expertos, especialistas y hombres con voluntad de expresarse aportan en menor proporción como fuentes opcionales y están más posicionados en la representación del poder oficial.

\begin{tabular}{cccc} 
Tipo de fuentes & Rechazan & Apoyan & Total \\
\hline Masculinas & 62 & 197 & 259 \\
& $18,67 \%$ & $25,3 \%$ & $23,5 \%$ \\
\hline \multirow{2}{*}{ Femeninas } & 109 & 89 & 198 \\
& $32,84 \%$ & $11,4 \%$ & $18 \%$ \\
\hline \multirow{2}{*}{ Documentos } & 161 & 492 & 653 \\
& $48,49 \%$ & $63,3 \%$ & $58,5 \%$ \\
\hline Total & $332(100 \%)$ & $778(100 \%)$ & $1.110(100 \%)$ \\
\hline
\end{tabular}

Tabla 5. Fuentes que aceptan y que rechazan la versión oficial

Fuente: Elaboración propia. 


\section{La perspectiva de los periódicos en casos de feminicidio en la prensa mexicana}

En esta parte se analiza la tercera premisa: las notas relacionadas con los casos de feminicidio en la prensa nacional mexicana disminuyen la perspectiva crítica. Los siguientes hallazgos están relacionados con la composición del texto. Posteriormente, se presentan aquellos ligados a las categorías, cuatro aspectos que sobresalen del análisis general acerca de los textos.

1. La mayoría de los textos se construye con una sola fuente. De las 2091, la concentración está en una fuente por texto, 858 (41\%); dos fuentes, 564 (27\%); tres fuentes, 342 (16\%); cuatro fuentes, 188 (9\%), y en menor proporción se localizan de cinco a 12 fuentes. Por periódico: La Jornada tuvo 530 fuentes (25,35\%); Reforma 341 (16,3\%); El Universal (663; 31,7\%), y Milenio (557; 26,65\%). Los textos con más de dos fuentes muestran una postura homogénea y no garantizan versiones que contrasten el hecho.

2. Los resultados también permiten afirmar que hay una reducida investigación periodística sobre el fenómeno del feminicidio. El tema estuvo presente en tanto que noticia en 1071 textos (81\%); el resumen con varios hechos implicó 177 textos (13,3\%); respecto de la opinión, hubo 63 textos (4,7\%); las entrevistas y gráficos independientes tuvieron nueve textos $(0,7 \%)$, y en el área de reportajes, únicamente Milenio publicó cuatro (0,3\%). El tipo de texto muestra que el feminicidio es abordado a partir de la nota diaria y en muy pocas ocasiones se profundiza en la problemática.

3. La mayor proporción de textos se construye gracias a los boletines. Los documentos o fuentes sin identificar ascendieron a 976 de 2091 fuentes (46,67\%), lo que significa que casi la mitad de los textos proviene de comunicados de prensa, estudios o fuentes sin identificar.

4. El porcentaje de reporteros es mayor que el de reporteras. La presencia de los primeros fue de 10\% más (tabla 2). De los 1324 textos, 547 textos fueron de reporteros (41\%), $408(31 \%)$ de reporteras, $47(4 \%)$ fueron escritos por un reportero y reportera y, hubo $322(24 \%)$ sin firma o con respaldo del periódico o agencia. Milenio fue el periódico que más recurrió a esa práctica.

Con relación al uso de las fuentes y los periódicos, los cuatro diarios consultaron mayor número de fuentes masculinas que aceptan la versión oficial por sobre las femeninas (tabla 6), mientras que el resultado acerca de las fuentes femeninas fue más heterogéneo por periódico. La Jornada tuvo un amplio margen entre las fuentes femeninas y masculinas que aceptan la versión oficial, habiendo más masculinas. Y también tuvo un amplio margen entre las fuentes femeninas que aceptan y rechazan la versión oficial, habiendo más fuentes femeninas que rechazan dicha versión. 


\begin{tabular}{c|cc|cc|cc|cc|c} 
Periódicos & \multicolumn{2}{|c|}{ La Jornada } & \multicolumn{2}{|c|}{ Reforma } & \multicolumn{2}{|c|}{ El Universal } & \multicolumn{2}{|c|}{ Milenio } & \\
\hline Fuentes & $\mathrm{A}$ & $\mathrm{R}$ & $\mathrm{A}$ & $\mathrm{R}$ & $\mathrm{A}$ & $\mathrm{R}$ & $\mathrm{A}$ & $\mathrm{R}$ & Total \\
\hline Masculina & 47 & 33 & 37 & 5 & 58 & 13 & 55 & 11 & 259 \\
\hline Femenina & 9 & 55 & 17 & 5 & 20 & 25 & 43 & 24 & 198 \\
\hline Documentos & 85 & 67 & 90 & 23 & 195 & 37 & 122 & 34 & 653 \\
\hline Total & 141 & 155 & 144 & 33 & 273 & 75 & 220 & 69 & 1110 \\
\hline
\end{tabular}

Nota: A es aceptan, R es rechazan.

Tabla 6. Tipo de fuentes por periódico que rechazan la versión oficial

Fuente: Elaboración propia.

Reforma y Milenio presentaron menor número de fuentes femeninas que rechazan la versión oficial, comparadas con las fuentes femeninas que aceptan la versión oficial.

\section{DISCUSIONES Y CONCLUSIONES}

Mediante diversos estudios, los investigadores muestran que en los medios de comunicación hay una tendencia a un mayor uso de fuentes masculinas (LenRíos, et al, 2005; Tamari et al., 2011; GMMP, 2021). Para Len-Ríos y sus colegas (2005), la identificación de ese patrón está relacionada con la hegemonía cultural masculina, mientras que los otros autores mencionan la desproporción que pone en desventaja social a la mujer. Un aspecto en el que difiere este trabajo de los estudios anteriores es que en los resultados generales de esta investigación las fuentes masculinas están menos presentes que las femeninas. Lo que aquí se identifica específicamente es el contraste en relación con la oficialidad de las fuentes: las oficiales masculinas son más que las femeninas. En particular, el grueso de fuentes masculinas se encuentra en la categoría de las que aceptan la versión oficial, lo que se traduce en una discriminación de fuentes oficiales femeninas en lo que concierne a un problema que atañe a mujeres, el feminicidio.

En relación con los resultados y propuestas de Gallur y García (2019) sobre el aspecto crítico de las fuentes, los hallazgos de esta investigación coinciden con lo registrado por los autores. En la categoría de las fuentes que rechazan la versión oficial, la presencia de voceras femeninas fue mayor; por ende, las voces masculinas tuvieron menor presencia, lo que sugiere que la postura crítica acerca del tema del feminicidio en la prensa mexicana la aportan las mujeres, que son quienes desde el sistema institucional son más exigentes y ponen en evidencia las debilidades de las políticas públicas y la forma en la que operan las instancias de justicia. 
Aunado a eso, la participación de fuentes masculinas también es menor como especialistas, expertos y personas con voluntad de expresarse, mientras que las fuentes femeninas son más amplias, lo que visibiliza que la discusión sobre el feminicidio se da a través de voceras que desde la sociedad civil aportan a la temática.

En la literatura se destaca que, en casos de feminicidio, quien construye el texto acude generalmente a fuentes oficiales y que hay una fuerte dependencia de documentos como los que emiten las dependencias de seguridad que proceden de instancias oficiales. Lagos (2008) las identificó como fuentes policiales, mientras que Taylor (2009) refiere a los testimonios que están en los reportes de las autoridades. Coincidimos con estos autores en que predominan las fuentes oficiales. Asimismo, este trabajo establece la proporción de documentos como boletines, comunicados, estudios y fuentes sin identificar que se utilizan al momento de redactar la noticia de un feminicidio; este tipo de fuentes abarca el mayor número de fuentes oficiales. Así, la prensa escrita mexicana analizada carece también de una perspectiva crítica, al replicar la información que les es proporcionada, y pierde la oportunidad de contrastar versiones. En ese sentido, lo que le llega al lector es la versión de las autoridades, en la que el medio presenta de manera parcial la información.

Se puede confirmar mediante esta investigación que la oficialidad de las fuentes informativas está directamente vinculada con el género masculino. Es decir, la mayor parte de las fuentes oficiales elegidas por los medios de comunicación estudiados son hombres. Esto afectaría directamente a los textos informativos en dos vertientes diferenciadas: por un lado, estaría la exclusión de las fuentes no oficiales y de género femenino y, por otro, la perspectiva crítica. Así, el nivel crítico del discurso de las fuentes utilizadas por el medio es inversamente proporcional al número de fuentes masculinas utilizadas, y a su nivel de oficialidad.

De lo anterior se podría inferir que, a mayor grado de oficialidad de los hombres consultados como fuentes, menor grado de crítica de dichas noticias (Gallur y García, 2019). Por ello, en esta investigación se ha constatado un elevado nivel de discriminación hacia las mujeres como fuentes de información oficial en el tema del feminicidio en México en los periódicos analizados, mediante una estructura tradicional y definida tanto del gatekeeping (Shoemaker \& Reese, 2014) como de la misma agenda setting (McCombs, 1972; 2006). Por lo tanto, el género es una categoría que los medios de comunicación usan en la discriminación de las fuentes válidas en la construcción del hecho noticioso.

\section{REFERENCIAS}

Álvarez-Monsiváis, E. (2020). Encuadres noticiosos sobre primeras damas: las expertas, las novatas y las actuales (Framing first ladies: the experts, the novices, and the incumbents). Profesional de la información, 29(6), e290621. https://doi.org/10.3145/epi.2020.nov.21 
Bautista, E. (2013). Las tendencias ideológico-políticas en el manejo de la información de los periódicos El Universal, Reforma y Milenio, en el caso de la detención de Gregorio Sánchez Martínez, candidato a la gubernatura de Quintana Roo en 2010 por la coalición PRD, PT y Convergencia (The ideological-political tendencies in the handling of the information of the newspapers El Universal, Reforma \& Milenio, in the case of the arrest of Gregorio Sánchez Martínez, candidate for governor of Quintana Roo in 2010 of the PRD, PT \& Convergencia coalition) (Undergraduate thesis, Universidad Autónoma de México). http://132.248.9.195/ptd2013/agosto/0698983/0698983.pdf

Bejarano, M. (2014). El feminicidio es sólo la punta del iceberg (Femicide is just the tip of the iceberg). Región y sociedad, 26(4), 13-44. https://doi.org/10.22198/rys.2014.0.a85

Bernal, A. L. D. \& Flores, J. A. (2018). Feminicidios y Políticas Públicas: declaratorias de alertas de violencia de género en México, 2015-2017 (Femicides and Public Policies: Declarations of Gender Violence Alerts in Mexico, 2015-2017). Perspectiva Geográfica: Revista del Programa de Estudios de Posgrado en Geografía, 23(2), 33-57. https://revistas.uptc.edu.co/index.php/perspectiva/article/view/7287

Berkowitz, D. A. (2009). Reporters and their sources. In K. Wahl-Jorgensen \& T. Hanitzsch (Eds.), The Handbook ofJournalism Studies (pp. 122-135). Routledge. https://doi.org/10.4324/9780203877685

Berkowitz, D. \& Liu, Z. (2014). The Social-Cultural Construction of News. From Doing Work to Making Meanings. In R. S. Fortner \& P. M. Fackler (Eds.), The Handbook of Media and Mass Communication Theory (pp. 301-313). Wiley Blackwell. https://doi.org/10.1002/9781118591178.ch17

Berlo, D. K. (1999). El proceso de la comunicación: introducción a la teoría y a la práctica (The communication process: Introduction to theory and practice). El Ateneo.

Bezunartea, O. (1988). Noticias e ideología profesional. Los periodistas vascos en la transición (News and professional ideology. Basque journalists in the transition). Ediciones Deusto.

Breed, W. (1958). Mass communication and socio-cultural integration. Social Forces, 37(2), 109-116. https://doi.org/10.2307/2572792

Bystrom, D. G. (2004). Women as political communication sources and audiences. In L. Lee Kaid (Ed.), Handbook of Political Communication Research (pp. 435-459). Routledge. https://doi.org/10.4324/9781410610584

Caputi, J. \& Russell, D. E. (1990). Femicide: Speaking the unspeakable. Ms, 1(2), 34-47.

Carpenter, S. (2008). How online citizen journalism publications and online newspapers utilize the objectivity standard and rely on external sources. Journalism $\mathcal{E}$ Mass Communication Quarterly, 85(3), 531-548. https://doi.org/10.1177/107769900808500304

Danés, S. (2017). La representación del feminicidio en la prensa regiomontana (The representation of femicide in the Monterrey press) (Master's thesis, Instituto Tecnológico y de Estudios Superiores de Monterrey). https://repositorio.itesm.mx/ortec/handle/11285/622529

Diezhandino, P. (Coord.). (2007). Periodismo en la era de Internet (Journalism in the Internet age). Ariel.

Durán, G. M. (2009). La Ideología en los medios: Una Propuesta para un Mapa Conceptual de la Prensa en México (Ideology in the media: A proposal for a concept map of the press in Mexico) (Undergraduate thesis, Instituto Tecnológico y de Estudios Superiores de Monterrey). http://hdl.handle.net/11285/572959 
Freedman, E., Fico, F., \& Love, B. (2004). Male and female sources in newspaper coverage of male and female candidates in US senate races in 2004. Journal of Women, Politics $\mathcal{E}$ Policy, 29(1), 57-76. https://doi.org/10.1300/j501v29n01_04

Gallur, S. \& García, B. (2019). El uso de las fuentes como estrategia de producción informativa en una agencia de noticias de género: El caso de CIMAC (The use of sources as an estrategy of news production in a gender news agency: The case of CIMAC). Estudios Sobre El Mensaje Periodístico, 25(3), 1421-1443. https://doi.org/10.5209/esmp.66995

Gans, H. J. (2004). Deciding what's news: A study of CBS evening news, NBC nightly news, Newsweek, and Time. Northwestern University Press.

Gómez, E. (2016). Culpabilización de las víctimas y reconocimiento: límites del discurso mediático sobre la violencia de género (Victim Blaming and Recognition: Boundaries of Media Discourse on Gender Violence). Feminismo/s, (27), 197-218. https://doi.org/10.14198/fem.2016.27.11

GMMP. (2021). Who Makes the News? Global Media Monitoring Project 2020. https://whomakesthenews.org/wp-content/uploads/2021/07/GMMP-2020.Highlights. spa_.FINAL_.pdf

Grijelmo, A. (2002). El estilo del periodista (The Journalist's Style). Santillana.

Herman, E. S. \& Chomsky, N. (1988). Manufacturing Consent: The Political Economy of the Mass Media. Pantheon Books.

Jasso, C. \& González, K. (2018, September 10). Brecha en las mediciones de feminicidio en México (Gap in Femicide Measurements in Mexico). Resonancias. https://www.iis.unam.mx/blog/brechas-en-la-medicion-de-feminicidios-en-mexico/

Lagos, C. (2008). El feminicidio según la prensa chilena: Otra forma de violencia contra las mujeres (Femicide according to the Chilean press: Another form of violence against women) (Master's thesis, Universidad de Chile). https://repositorio.uchile.cl/handle/2250/106063

Len-Ríos, M. E., Rodgers, S., Thorson, E., \& Yoon, D. (2005). Representation of Women in News and Photos: Comparing Content to Perceptions. Journal of Communication, 55(1), 152-168. https://doi.org/10.1111/j.1460-2466.2005.tb02664.x

Ley General de Acceso de las Mujeres a una Vida Libre de Violencia (General Law on Women's Access to a Life Free of Violence) (2007, February 1). http://www.diputados.gob.mx/LeyesBiblio/ref/lgamvlv/LGAMVLV_orig_01feb07.pdf

Ley General para la Igualdad entre Mujeres y Hombres (General Law for equality between Women and Man) (2006, August 2). http://www.diputados.gob.mx/LeyesBiblio/pdf mov/Ley_General_para_la_Igualdad_entre_Mujeres_y_Hombres.pdf

Lippmann, W. (1965). Public Opinion. Free Press.

Lozano, J. C. (2007). Teoría e investigación de comunicación de masas (Theory and research of mass communication). Pearson Comunicación.

Manning, P. (2013). Financial journalism, news sources and the banking crisis. Journalism, 14(2), 173-189. https://doi.org/10.1177/1464884912448915

McCombs, M. (2006). Establecimiento de la agenda: el impacto de los medios en la opinión pública y en el conocimiento (Establishment of the agenda setting: the impact of the media on public opinion and knowledge). Grupo Planeta (GBS). 
McCombs, M. E. \& Shaw, D. L. (1972). The agenda-setting function of mass media. Public opinion quarterly, 36(2), 176-187. http://doi.org./10.1086/267990

Menéndez, M. I. (2014). Retos periodísticos ante la violencia de género: El caso de la prensa local en España (Journalistic challenges in relation to gender violence. The case of the Spanish local press). Comunicación y sociedad, (22), 53-77. https://doi.org/10.32870/cys.v0i22.48

Monárrez, J. E. (2010). Las diversas representaciones del feminicidio y los asesinatos de mujeres en Ciudad Juárez, 1993-2005 (The various representations of feminicide and murders of women in Ciudad Juárez, 1993-2005). In J. E. Monárrez, L. E. Cervera, C. M. Fuentes, \& R. Rubio (Coords.), Violencia contra las mujeres e inseguridad ciudadana en Ciudad Juárez (pp. 361-394). El Colegio de la Frontera Norte.

Monárrez, J. E. (2019). Feminicidio sexual sistémico: impunidad histórica constante en Ciudad Juárez, víctimas y perpetradores (Systemic Sexual Feminicide: a constant historical impunity in Ciudad Juárez, victims and perpetrators). Estado \& comunes, revista de políticas $y$ problemas públicos, 1(8). https://doi.org/10.37228/estado_comunes.v1.n8.2019.99

Niemi, M. \& Pitkanen, V. (2016). Gendered use of experts in the media: Analysis of the gender gap in Finnish news journalism. Public Understanding of Science, 26(3), 355-368. https://doi.org/10.1177/0963662515621470

Ortega, F. \& Humanes, L. (2000). Algo más que periodistas (More than journalists). Ariel, SA.

Pew Research Center. (2005). The Gender Gap. Women Are Still Missing as Sources for Journalists. Pew Research Center. https://pewrsr.ch/37HBBaW

Powers, A. \& Fico, F. (1994). Influences on use of sources at large US newspapers. Newspaper Research Journal, 15(4), 87-97. https://doi.org/10.1177/073953299401500410

Radford, J. \& Russell, D. E. (1992). Femicide: The politics of woman killing. Twayne Publishers.

Rizo, L. A. (2006). ¿A qué llamamos exclusión social? (What do we call social exclusión?) Polis. Revista Latinoamericana, 15. http://journals.openedition.org/polis/5007

Rodelo, F. V. \& Muñiz, C. (2017). La orientación política del periódico y su influencia en la presencia de encuadres y asuntos dentro de las noticias (The political orientation of the newspaper and its influence on the presence of frames and issues within the news). Estudios sobre el Mensaje Periodístico, 23(1), 241-257. https://doi.org/10.5209/esmp.55594

Retegui, L. (2017). La construcción de la noticia desde el lugar del emisor. Una revisión del newsmaking (The Process of Making News from the Newsmaker's Perspective. A Review of Newsmaking). Revista mexicana de opinión pública, (23), 103-121. https://doi.org/10.22201/fcpys.24484911e.2017.23.56354

Salgado, E. (2005). Una nueva tipología para analizar la prensa en México (A new typology to analyze the press in Mexico). Revista latinoamericana de estudios del discurso, 5(2), 107-125. http://raled.comunidadaled.org/index.php/raled/article/view/148/150

Salazar, M. G., Bravo, C., Vaca, M., Paxman, A., López, A., \& Hughes, S. (2019). Democracia, prensa y poder en México: un debate sobre Newsrooms in Conflict, de Sallie Hughes (Democracy, the Press and the Power in Mexico: A Debate on Newsrooms in Conflict, by Sallie Hughes). Politica y gobierno, 26(2). http://www.politicaygobierno.cide.edu/index.php/pyg/article/view/1301 
Salazar, M. G. \& de la Garza, P. (2020). La cobertura periodística de los feminicidios en México. Heterogeneidad y variación (Journalistic coverage of femicides in Mexico. Heterogeneity and variation). Revista mexicana de opinión pública, (29), 111-125. https://doi.org/10.22201/fcpys.24484911e.2020.29.70633

Secretariado Ejecutivo del Sistema Nacional de Seguridad. (2021, October 31). Información sobre la violencia contra las mujeres. víctimas de homicidio, secuestro y extorsión. Incidencia delictiva y llamadas de emergencia al 9-1-1 (Information on violence against women. victims of homicide, kidnapping and extortion. Crime Incidence and 9-1-1 Emergency Calls). SESNSP-Gobierno de México.

Shoemaker, P. J. \& Reese, S. D. (2014). Mediating the message in the 21st century: Theories of influence on mass media content. Routledge.

Sigal, L. V. (1986). Sources make the news. In R. K. Manoff \& M. Schudson (Eds.), Reading the news (pp. 9-37). Pantheon.

Tagle Martínez, M. A. (2017). Vida libre de violencia: Un derecho pendiente para las mujeres (Life free of violence: A pending right for women). Pluralidad $y$ Consenso, 7(31), 100-109. http://revista.ibd.senado.gob.mx/index.php/PluralidadyConsenso/article/view/404

Tamarit, A., Paz, N. Q., \& Plaza, J. F. (2011). ¿Quién habla de las mujeres en las noticias donde ellas son las protagonistas? (Who Talks About Women in the News in Which They Are Protagonists?). Palabras Clave, 14(2), 247-260. https://doi.org/10.5294/pacla.2011.14.2.4

Taylor, R. (2009). Slain and slandered. A content analysis of the portrayal of femicide in crime news. Homicide Studies, 13(1), 21-49. https://doi.org/10.1177/1088767908326679

Tuchman, G. (1973). Making news by doing work: Routinizing the unexpected. American journal of Sociology, 79(1), 110-131. https://www.journals.uchicago.edu/doi/abs/10.1086/225510

Tiscareño-García, E. (2021). El lenguaje en la prensa escrita mexicana sobre víctimas $\mathrm{y}$ victimarios o presuntos victimarios de feminicidio (The language in the Mexican written press about victims and perpetrators or alleged perpetrators of femicide) (Doctoral dissertation, Instituto Tecnológico y de Estudios Superiores de Monterrey). https://repositorio.tec.mx/handle/11285/637313

Tiscareño-García, E. \& Miranda-Villanueva, O. (2020). Victims and perpetrators of feminicide in the language of the Mexican written press. Comunicar, 63, 51-60. https://doi.org/10.3916/C63-2020-05

Van der Pas, D. J. \& Aaldering, L. (2020). Gender Differences in Political Media Coverage: A Meta-Analysis. Journal of Communication, 70(1), 114-143. https://doi.org/10.1093/joc/jqz046

Wimmer, R. \& Dominick, J. (2011). Part two. Research approaches. In R. Wimmer \& J. Dominick (Eds.), Mass Media Research (pp. 114-148). Wadswort Cengage Learning.

Xantomila, Y. (2020, March 5). ONU: Feminicidios en México crecieron diariamente de 7 a 10 en tres años (Femicides in Mexico grew daily from 7 to 10 in three years). La Jornada. https://www.jornada.com.mx/ultimas/sociedad/2020/03/05/onu-feminicidios-enmexico-crecieron-de-7-a-10-diarios-en-tres-anos-8647.html 


\section{SOBRE LOS AUTORES}

ELIZABETH TISCAREÑo-GARCía, Investigadora de la Escuela de Educación y Humanidades del Tecnológico de Monterrey. Su tesis doctoral estudió el lenguaje sobre víctimas y victimarios o presuntos victimarios de feminicidio en la prensa escrita mexicana. Tiene una maestría en Análisis político y Medios de Información (EGAP, Tecnológico de Monterrey). Es periodista egresada de la Facultad de Ciencias de la Comunicación (Universidad Autónoma de Nueva León, México). Se ha desempeñado en televisión, radio y páginas web como responsable de departamentos de noticias en México. Se especializa en estudios sobre framing en casos de juicios mediáticos (caso de Florence Cassez), migración y feminicidio.

iD http://orcid.org/0000-0002-5465-2472

SANTIAGo GALlUR-SANTORUM, Profesor pleno del Instituto Tecnológico de Santo Domingo (INTEC, República dominicana), Doctor en Historia Contemporánea y de América Latina (2011, USC, España), Doctor en Comunicación e Información (2017, USC, España), Maestro en Historia Contemporánea y de América Latina (2009, USC, España), Maestro en Periodismo (2017, España). Miembro del Sistema Nacional de Investigadores de México, SNI 1. Líneas de investigación: clickbait, fake news, el género en los medios de comunicación, la difusión del feminicidio y las consecuencias de la violencia en la información.

http://orcid.org/0000-0001-6287-7340

OSCAR MARIO MIRANDA-VILLANUEVA, Profesor Investigador de la Escuela de Humanidades y Educación (EHE), del Tecnológico de Monterrey. Es Investigador Nacional Nivel I, por el Sistema Nacional de Investigadores (SNI- CONACYT), en México. Ha sido parte de equipos de investigación con proyectos financiados por CONACYT y UC Mexus, en conjunto con la Universidad Regiomontana, la Universidad de California en Davis y Berkeley. Las temáticas que cubren sus publicaciones gravitan en las distintas dimensiones de los estudios de audiencias.

iD http://orcid.org/000-0003-3180-889X 